\title{
Salinidad, composición botánica y crecimiento de especies frutales en huertos familiares de Tabasco, México
}

\section{Salinity, botanical composition and growth of fruit species in home gardens in Tabasco, Mexico}

\author{
Alejandro Alcudia-Aguilar ${ }^{1}$, Hans van der Wal ${ }^{1 *}$, Juan Suárez-Sánchez ${ }^{2}$, David Álvarez-Solis ${ }^{3}$, Cristian \\ Tovilla-Hernández ${ }^{4}$ \\ ${ }^{1}$ Departamento de Agricultura Sociedad y Ambiente, El Colegio de la Frontera Sur, Unidad Villahermosa. Carretera \\ Villahermosa a Reforma Km. 15.5, Ra. Guineo Segunda Sección. CP. 86280, Villahermosa, Centro, Tabasco, México. \\ ${ }^{2}$ Laboratorio de Medio Ambiente, Facultad de Agrobiología, Universidad Autónoma de Tlaxcala. \\ ${ }^{3}$ El Colegio de la Frontera Sur, Unidad San Cristóbal de las Casas. \\ ${ }^{4}$ El Colegio de la Frontera Sur, Unidad Tapachula. \\ *Autor de correspondencia: hvanderwal@ecosur.mx
}

Artículo científico recibido: 26 de octubre de 2014, aceptado: 28 de enero de 2016

RESUMEN. El presente trabajo tiene como objetivo determinar si la salinidad del suelo incide en la composición de especies arbóreas y arbustivas en los huertos familiares de la costa del estado de Tabasco, México, y si la tolerancia a la salinidad varía entre las tres especies más frecuentes en el área. Se determinó la frecuencia y abundancia de especies arbóreas y arbustivas en 19 huertos familiares, y se determinó la conductividad eléctrica (CE) del suelo. En vivero se sometieron plantas de guanábana (Annona muricata L.), mango (Mangifera indica L.) y naranja agria (Citrus aurantium L.) a CE de 124, 1 084, 2143,4031 y $7631 \mu \mathrm{S} \mathrm{cm}^{-1}$ en el agua de riego, para luego determinar la altura de las plantas y la biomasa de raíz, tallo y hojas. Se calculó la CE a la que se reduce la biomasa en $50 \%\left(\mathrm{CE}_{50}\right)$ y el índice de tolerancia al estrés (ST-Index). La composición de especies en campo cambió con la salinidad de los suelos. La $\mathrm{CE}_{50}$ en guanábana, mango y naranja agria fueron de 9 580, 10220 y $17430 \mu \mathrm{S} \mathrm{cm}^{-1}$, y el ST-Index fue de 16 810, 9300 y $8830 \mu \mathrm{S} \mathrm{cm}^{-1}$. La salinidad influye en la composición de especies. Las especies más frecuentes difieren en tolerancia al estrés, por lo que su composición en los huertos familiares cambiará al aumentar la salinidad del suelo por el cambio climático en la región.

Palabras clave: Î́ndice de tolerancia al estrés, guanábana, naranja agría, mango

ABSTRACT. The aim of this work was to determine if soil salinity influences the species composition of trees and shrubs in home gardens on the coast of the state of Tabasco, Mexico, and if the tolerance to salinity varies among the three most common species in the area. We determined the frequency and abundance of the tree and shrub species in 19 home gardens, as well as the electrical conductivity (EC) of the soil. In a nursery we subjected plants of the three most common species, namely soursop (Annona muricata L.), mango (Mangifera indica L.) and bitter orange (Citrus aurantium L.), to EC 124, $1084,2143,4031$ and $7631 \mu \mathrm{S} \mathrm{cm}^{-1}$ in irrigation water, and afterwards plant height and root, stem and leaf biomass were determined. We calculated the $\mathrm{EC}$ that reduces biomass by $50 \%\left(\mathrm{EC}_{50}\right)$, and the stress tolerance index (ST-Index). The species composition in the field changed with soil salinity. The $\mathrm{EC}_{50}$ in soursop, mango and sour orange was 9 580, 10220 and $17430 \mu \mathrm{S} \mathrm{cm}^{-1}$, and the ST-Index was 16810,9300 and $8830 \mu \mathrm{S}$ $\mathrm{cm}^{-1}$. Salinity influences species composition, and the most common species showed different tolerance to stress, so the species composition in home gardens will change as soil salinity increases through the impact of climate change in the region.

Key words: Stress tolerance index, soursop, mango, orange 


\section{INTRODUCCIÓN}

Los huertos familiares son asociaciones adaptadas de árboles, arbustos, cultivos herbáceos y/o animales, manejados con base en la mano de obra familiar (Galluzzi et al. 2010). Se encuentran en comunidades rurales, alcanzando su mayor biodiversidad y riqueza estructural en climas tropicales (Kumar y Nair 2004). Cumplen con un conjunto de funciones ecológicas, ya que conservan biodiversidad, promueven el reciclaje de nutrientes, evitan la erosión, generan beneficios hidrológicos, proveen nichos de aves, y crean micro-climas (Gliessman 1998). Además de proveer múltiples beneficios económicos, al generar productos. Su diversidad de especies vegetales y animales permite obtener productos alimenticios para autoconsumo y venta durante todo el año, además de leña, medicinas, condimentos y ornamentos (Idohou et al. 2014). La composición de las especies en los huertos familiares tiene relación con las condiciones climáticas y edáficas, al cultivarse las especies que mejor se adaptan a ellas (Pitman y Läuchli 2002). Sin embargo, también se adaptan estas condiciones a los requerimientos de las especies, cultivando plantas en recipientes y aplicando riegos (Nakashima 2012).

El cambio climático modifica las condiciones de los suelos y clima en los huertos, particularmente en las costas, y pueden influir en la composición de especies de los huertos. Ya que el incremento del nivel del mar provoca aumento de la salinidad (Caetano et al. 2010). También el cambio climático tiende a incrementar la frecuencia e intensidad de eventos hidrometeorológicos extremos, que afectan los ecosistemas (Ortíz-Pérez et al. 2010). La salinidad del suelo afecta la capacidad de las plantas para absorber agua y regular su temperatura; al volver inhóspito al suelo, afecta también la distribución de las raíces, lo que ocasiona deficiencias de nutrimentos y desórdenes fisiológicos en las plantas (Xu et al. 2014).

Elevadas concentraciones de $\mathrm{Na}^{+}$y $\mathrm{Cl}^{-}$en el suelo disminuyen las actividades de otros iones, conduciendo a desequilibrios nutricionales de $\mathrm{Ca}^{2+}$, $\mathrm{K}^{+}, \mathrm{Mg}^{2+}$ y $\mathrm{NO}_{3}-$, y reducen la disponibilidad y transporte de $\mathrm{Ca}^{2+}$ a las partes en crecimiento de la planta (Kopittke et al. 2011). La salinidad elevada afecta el desarrollo de las hojas y el crecimiento del tallo (Zhu et al. 2012). Las especies toleran distintas concentraciones de sal en el entorno antes de verse afectadas de forma negativa (Chaitanya et al. 2014). La tolerancia a la salinidad se expresa en términos de biomasa relativa, esto es, la relación entre la biomasa generada a determinada concentración de sal y la biomasa generada sin salinidad: $Y_{r}=Y / Y_{m}$. Donde, $\mathrm{Y}_{r}$ es la biomasa relativa, $Y$ la biomasa obtenida con salinidad, e $Y_{m}$ es la biomasa obtenida sin salinidad. Mientras que Correia et al. (2010) ajustaron un modelo exponencial que relaciona la biomasa relativa del algarrobo (Ceratonia siliqua L.) con la CE del agua de riego, obteniendo la ecuación $Y_{r}=a e^{b E C}$. Donde, $\mathrm{ECi}$ es la $\mathrm{CE}_{i}$ del agua de riego, y a y b son constantes del modelo. Mientras que Steppuhn et al. (2005a), a partir de la relación entre las concentraciones de sales y la biomasa de las especies, propusieron el índice de tolerancia de estrés (ST-Index): $S T-$ index $=$ $E C_{i 50}(1+b)$. Donde, $E C_{i 50}$ es la $C E$ a la cual $Y_{r}$ (biomasa relativa) es igual a 0.5 , y $b$ la constante de la ecuación de Correia et al. (2010).

El objetivo fue analizar el efecto de la salinidad en la composición de especies en huertos familiares de la costa del estado de Tabasco, México, además de determinar si las tres especies más frecuentes en los huertos son tolerantes a los niveles de salinidad que se presentan en el suelo.

\section{MATERIALES Y MÉTODOS}

\section{Muestreo en huertos}

Se realizó un recorrido por ocho comunidades de la costa de los municipios de Paraíso $\left(18^{\circ} 16\right.$ LN $93^{\circ} 32$ LO), Jalpa de Méndez ( $18^{\circ} 04$ LN $93^{\circ} 00$ LO) y Comalcalco (18 09 LN 93 05 LO) (Figura 1), donde se reportan altas concentraciones de sal en el manto freático y el suelo (Caetano et al. 2010, Ortíz-Pérez et al. 2010). En los que seleccionaron 19 huertos, en los cuales se registraron los árboles y arbustos.

En cada huerto, se tomaron tres submuestras 


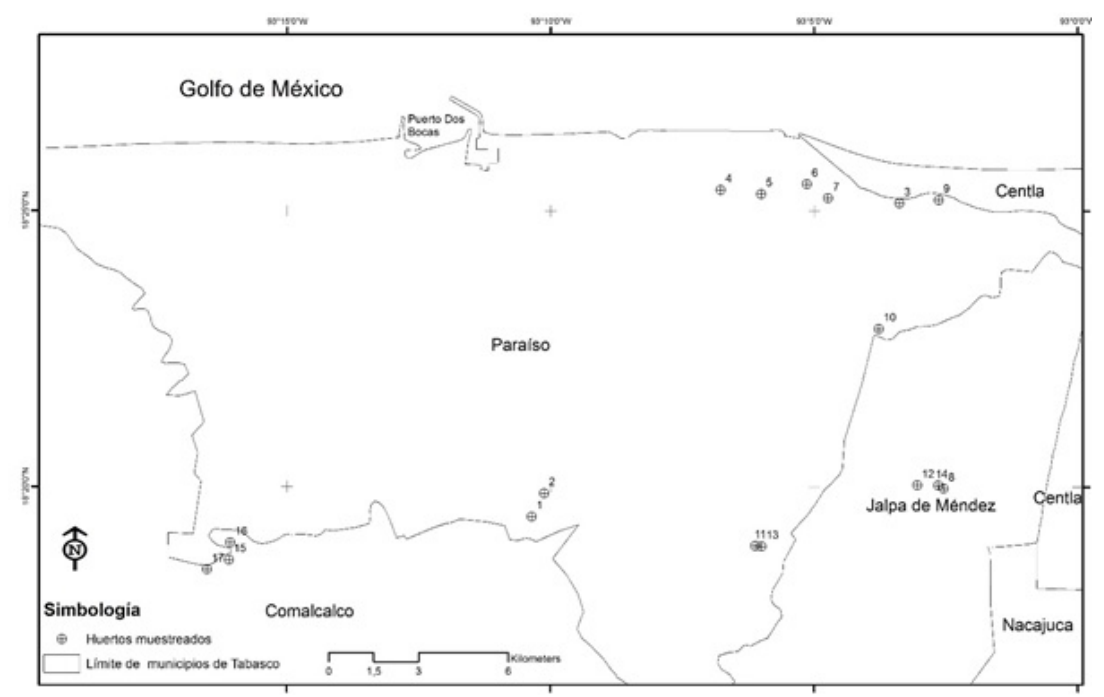

Figura 1. Localización de los huertos familiares de muestreo en la costa de Tabasco, México.

de las capas de 0 a 10,10 a 20 y 20 a $30 \mathrm{~cm}$ de profundidad del pedón, distribuidas sobre el área del huerto y mezcladas por cada capa en un recipiente para obtener una muestra compuesta, a finales de la temporada de lluvias (febrero y marzo), nortes (octubre, noviembre y diciembre), secas (marzo, abril y mayo) y lluvias (junio, julio, agosto y septiembre). Para lo cual se perforó el suelo con una barrena manual y se determinó la profundidad a la que se encontraba el manto freático. Las muestras de suelo se secaron en el laboratorio para luego determinar la CE en una submuestra de $12 \mathrm{~g}$ con el equipo multi-paramétrico Hannah modelo HI 9828.

En cada huerto se registraron las especies por su nombre común; cuando se encontraron especies desconocidas, se tomaron muestras para su identificación en el herbario de la Universidad Juárez Autónoma de Tabasco y El Colegio de la Frontera Sur, Unidad San Cristóbal de las Casas. Con los registros de las especies por huerto se generó una matriz de abundancia a la que se le aplicó el análisis canónico de correspondencia (ACC), con la CE y la profundidad del manto freático como variables ambientales.

\section{Experimento en vivero}

En un vivero con cubierta de malla sombra del $50 \%$ y plástico transparente, se realizó un experimento en el cual se aplicaba agua a distintas concentraciones de sal $(\mathrm{NaCl})$ a plantas de las tres especies más frecuentes en los huertos: guanábana (Annona muricata), mango (Mangifera indica) y naranja agria (Citrus aurantium), excluyendo el coco (Cocos nucifera) por presentar alta tolerancia a la salinidad (Remison et al. 1988). Las plantas de estas especies se adquirieron en un vivero comercial, procurando que fueran vigorosas, sanas, de la misma edad (aproximadamente ocho meses), de pie franco, y cultivadas en bolsas de volumen y sustrato similar. Las bolsas con las plantas fueron colocadas sobre estructuras de metal para evitar el contacto directo con el suelo y el enraizamiento. El experimento se realizó en El Colegio de la Frontera Sur, Unidad Villahermosa, del 19 de marzo al 19 de junio del 2012, periodo similar al evaluado por Correia et al. (2010). Durante el experimento la temperatura mínima media fue de $18.1^{\circ} \mathrm{C}$ y la temperatura media máxima de $33.9{ }^{\circ} \mathrm{C}$ (CONAGUA 2012).

\section{Diseño experimental}

En el experimento se utilizó un diseño de bloques al azar, siendo los tratamientos el agua de purificada (testigo, CE $124 \mu \mathrm{S} \mathrm{cm} \mathrm{cm}^{-1}$ ), $10 \mathrm{mmol}$ $\mathrm{NaCl} \mathrm{L}{ }^{-1}$ (CE $1084 \mu \mathrm{S} \mathrm{cm}{ }^{-1}$ ), $20 \mathrm{mmol} \mathrm{NaCl}$ 
Tabla 1. Cantidades acumuladas de sal aplicada a macetas con plantas de guanábana, naranja agria y mango.

\begin{tabular}{ccccc}
\hline Tratamiento & Concentración de NaCl & Fecha 1 $(\mathrm{g})$ & Fecha 2(g) & Fecha 3 $(\mathrm{g})$ \\
\hline 1 & Testigo & 0.00 & 0.00 & 0.00 \\
2 & $10 \mathrm{mmol} \mathrm{NaCl} \mathrm{L}^{-1}$ & 0.61 & 1.19 & 1.78 \\
3 & $20 \mathrm{mmol} \mathrm{NaCl} \mathrm{L}^{-1}$ & 1.22 & 2.37 & 3.56 \\
4 & $40 \mathrm{mmol} \mathrm{NaCl} \mathrm{L}^{-1}$ & 2.46 & 4.77 & 7.15 \\
5 & $80 \mathrm{mmol} \mathrm{NaCl} \mathrm{L}^{-1}$ & 4.93 & 9.55 & 14.33 \\
\hline Fecha 1: 32 d, Fecha 2: 62 d, Fecha 3: 93 d.
\end{tabular}

$\mathrm{L}^{-1}$ (CE $2143 \mu \mathrm{S} \mathrm{cm}^{-1}$ ), $40 \mathrm{mmol} \mathrm{NaCl} \mathrm{L}{ }^{-1}$ (CE $4031 \mu \mathrm{S} \mathrm{cm}^{-1}$ ) y $80 \mathrm{mmol} \mathrm{NaCl} \mathrm{L}^{-1}$ (CE $7631 \mu \mathrm{S}$ $\mathrm{cm}^{-1}$ ). Se utilizaron 25 plantas por especie en cada tratamiento, en las que se aplicaron cada tres d 300 $\mathrm{mL}$ de cada tratamiento.

\section{Evaluación de variables}

Se midió el crecimiento por medio de la altura de planta y el número de hojas, y la biomasa de hojas, tallos y raíces, cosechando al azar cinco plantas de cada uno de los tratamientos a los 32 d (fecha 1), a los $62 \mathrm{~d}$ (fecha 2), y a los $93 \mathrm{~d}$ (fecha 3) (Tabla 1). En la fecha dos, se midió la CE por duplicado en el agua sobrenadante en dos muestras compuestas del suelo de las cinco macetas cosechadas de cada tratamiento. En la fecha 3, después de secar las muestras hasta peso constante en una estufa a $60^{\circ} \mathrm{C}$, se realizaron tres mediciones de la CE del suelo en las macetas de cada uno de los tratamientos, realizando una medición en la mitad superior del suelo de la maceta; una en la mitad inferior, y una de la mezcla de las dos partes. La CE se midió utilizando el método de conductimetría (Etchevers 1992), añadiendo agua destilada al suelo en proporción 5: 1, para luego agitar la mezcla, dejarla reposar por $24 \mathrm{~h}$ y medir la CE. También se determinó el peso seco de las hojas, tallo y raíces, después de secar las plantas hasta peso constante en una estufa a $60^{\circ} \mathrm{C}$. El peso seco total (PST) se calculó con la suma de los pesos hojas, brotes, tallos y raíces. Para luego calcular la relación del peso de las hojas con el peso total de la planta (RPH); la relación peso de los tallos con el peso total de la planta (RPT) y la relación del peso de la raíz con el peso total de la planta (RPR).
Modelo de respuesta a la salinidad y ST-Index Se utilizó el modelo propuesto por Steppuhn et al. (2005) para modelar la respuesta de la biomasa total de las plantas en función de la conductividad del agua de riego y determinar la tolerancia a la salinidad de las tres especies.

\section{Análisis estadísticos}

Se realizó la prueba de Shapiro-Wilk para el análisis de normalidad. En caso de normalidad, se aplicó un ANOVA de una vía, con los tratamientos de salinidad como factores y las plantas como repeticiones. Cuando el valor de $\mathrm{F}$ fue significativo, se aplicó la prueba HSD de Tukey para determinar diferencias significativas entre pares de tratamientos. Cuando las variables no presentaron normalidad se usaron las pruebas no-paramétricas de KruskalWallis y Mann-Whitney. Después del ACC, se utilizó la prueba de permutación para determinar si el eigenvalue era significativo. Todos los análisis se realizaron en el programa PAST (Hammer et al. 2001).

\section{RESULTADOS}

\section{Biodiversidad y composición de especies}

Las especies frutales son las más frecuentes en los huertos. La palma de coco es la más abundante, posiblemente por ser tolerante a las altas concentraciones de sal. Después de la palma de coco, las plantas más frecuentes fueron la guanábana $(A$. muricata), el mango ( $M$. indica), la naranja agria (C. aurantium), el macuilis (Tabebuia rosea DC.), la guayaba (Psidium guajava L.), el limón (Citrus limon L. (Osbeck)) y la ciruela (Spondias purpurea L.). También se encuentran especies maderables, 


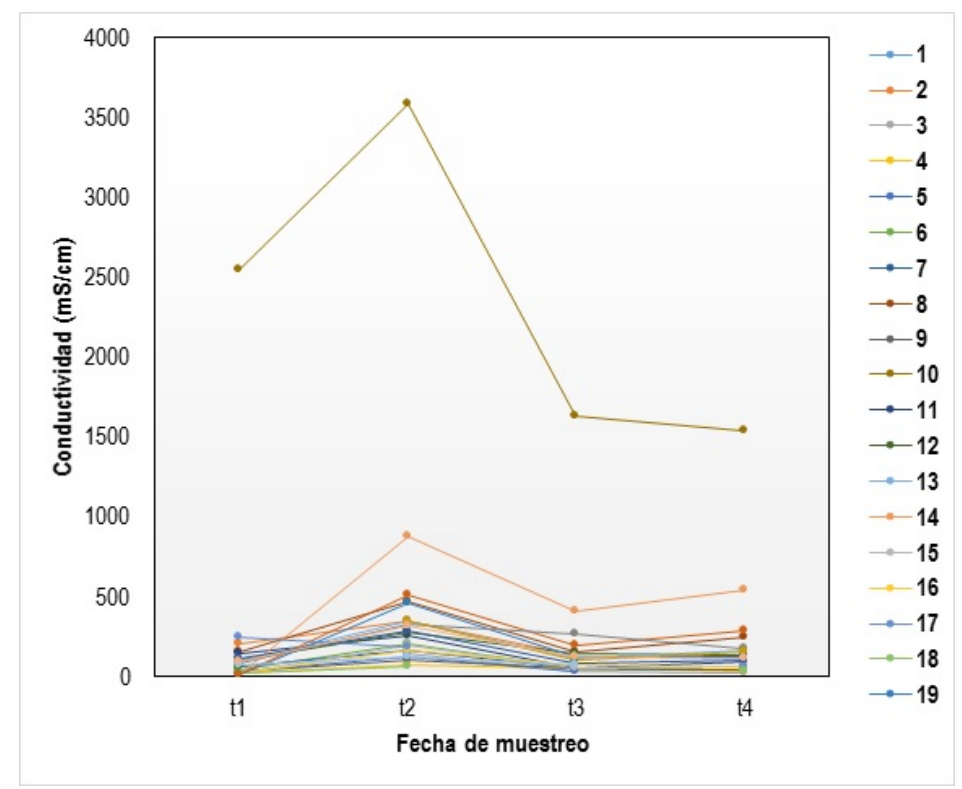

Figura 2. CE en los $30 \mathrm{~cm}$ superiores del suelo en huertos familiares en la costa de Tabasco, México, al inicio de la temporada de nortes, inicio y final de secas, y lluvias (t1 = diciembre 2011, $\mathrm{t} 2=$ marzo 2012, $\mathrm{t} 3=$ mayo 2012, t $4=$ julio 2012). Números del 1 al 19 indican los huertos evaluados.

como el cedro (Cedrela odorata L.) y forrajeras como el cocoíte (Gliricidia sepium Kunth).

\section{Variables del suelo y agua de los huertos}

La CE en la capa superior del suelo mostró variación entre los huertos y las temporadas de muestreo (Figura 2). La profundidad a la cual se encontró el agua freática fue entre 1.50 y $5.83 \mathrm{~m}$ (Tabla 2). La mayor profundidad se observó en el mes de mayo, al final de la temporada seca, mientras que la menor profundidad fue al final de la temporada de lluvias en el mes de diciembre $(\mathrm{F}=2.922$, $p=0.043$, Tukey HSD, $Q=4.096, p=0.028)$.

El ACC tuvo un eigenvalue de 0.288 en el eje 1 (Figura 3). Este valor es significativo de acuerdo con la prueba de permutación $(p=0.01)$. Las coordenadas de los huertos sobre el eje 1 mostraron una correlación significativa con los valores de CE (Spearman rho $=0.83, \mathrm{p}<0.001$ ), mientras que la correlación entre la profundidad del manto freático y las coordenadas en el eje 2 no fue significativa.
Tabla 2. Profundidad del agua freática en nortes, secas y lluvias en huertos familiares de la costa de Tabasco, México.

\begin{tabular}{lccc}
\hline & Promedio $(\mathrm{m})$ & Mínimo $(\mathrm{m})$ & Máximo $(\mathrm{m})$ \\
\hline Diciembre 2011 & 3.21 & 1.78 & 5.27 \\
Marzo 2012 & 3.41 & 2.00 & 5.75 \\
Mayo 2012 & 3.64 & 2.00 & 5.83 \\
Julio 2012 & 3.47 & 1.50 & 5.50 \\
\hline
\end{tabular}

\section{Salinidad del suelo en macetas}

En la fecha 2 (19 de mayo), la CE de las macetas se incrementó al al aumentar la concentración de sal en el agua de riego aplicada. Esto también, se observó en mango a los 93 d después del inicio del experimento, mientras que en guanábana se presentó la mayor conductividad en donde se aplicó la concentración de sal de $40 \mathrm{mmol} \mathrm{NaCl}$ $\mathrm{L}^{-1}$. En las macetas con naranja agria se observaron los niveles más altos de CE en el tratamiento con $80 \mathrm{mmol} \mathrm{NaCl} \mathrm{L}{ }^{-1}$ y alta $\mathrm{CE}$ en el tratamiento con $20 \mathrm{mmol} \mathrm{NaCl} \mathrm{L}^{-1}$ (Tabla 3). La CE en el suelo en la mitad superior de las macetas fue menor que en la parte inferior (Tabla 4), observándose acumulación de sal en la parte inferior. 


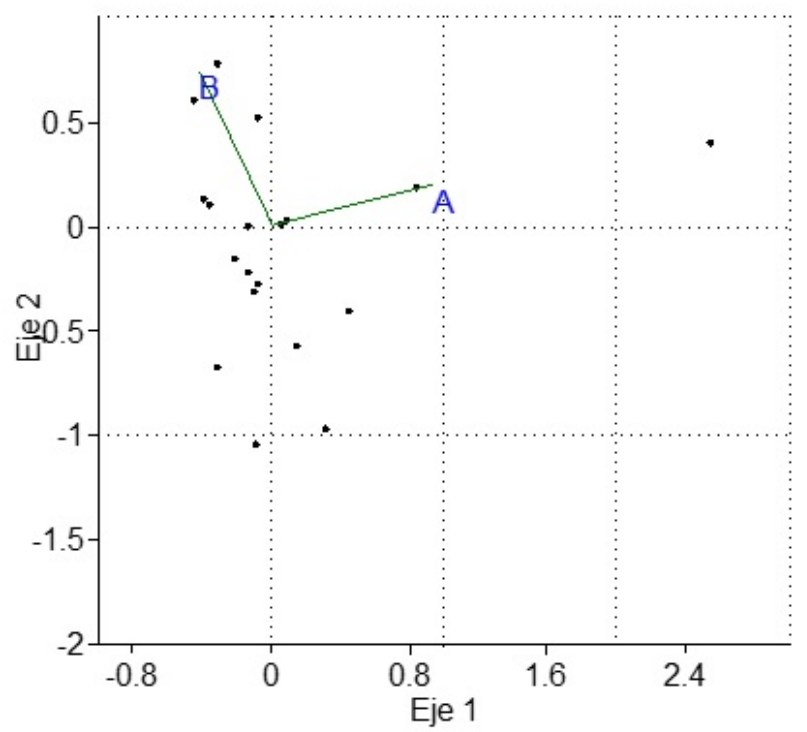

Figura 3. Análisis Canónico de Correspondencia con datos de abundancia de especies arbóreas y arbustivas de 19 huertos de la costa del estado de Tabasco, México.

Tabla 3. CE del suelo en las macetas con plantas de guanábana, mango y naranja.

\begin{tabular}{lrrrrrrrr}
\hline & \multicolumn{3}{c}{$62 \mathrm{~d}$} & & \multicolumn{3}{c}{$93 \mathrm{~d}$} \\
\cline { 2 - 4 } \cline { 8 - 9 } Tratamiento & Guanábana & Mango & Naranja & & Guanábana & Mango & Naranja \\
\hline Testigo & 93 & 81 & 210 & & 109 & 117 & 206 \\
$10 \mathrm{mmol} \mathrm{NaCl} \mathrm{L}^{-1}$ & 553 & 310 & 503 & & 825 & 624 & 711 \\
$20 \mathrm{mmol} \mathrm{NaCl} \mathrm{L}^{-1}$ & 723 & 729 & 1074 & & 943 & 1183 & 1367 \\
$40 \mathrm{mmol} \mathrm{NaCl} \mathrm{L}^{-1}$ & 1114 & 1363 & 1257 & & 2125 & 1926 & 1152 \\
$80 \mathrm{mmol} \mathrm{NaCl} \mathrm{L}^{-1}$ & 1356 & 1941 & 1888 & & 1452 & 2204 & 1514 \\
\hline
\end{tabular}

Tabla 4. CE del suelo en la mitad superior e inferior de macetas con plantas de guanábana, mango y naranja agria.

\begin{tabular}{|c|c|c|c|c|c|c|}
\hline \multirow{3}{*}{ Tratamiento } & \multicolumn{6}{|c|}{ Especies } \\
\hline & Guanábana & Mango & Naranja & Guanábana & Mango & Naranja \\
\hline & \multicolumn{3}{|c|}{ Mitad inferior } & \multicolumn{3}{|c|}{ Mitad superior } \\
\hline Testigo & $298.0^{a}$ & $228.0^{a}$ & $698.2^{a}$ & $59.0^{a}$ & $54.4^{a}$ & $75.8^{a}$ \\
\hline $10 \mathrm{mmol} \mathrm{NaCl} \mathrm{L}-1$ & $1555.0^{a}$ & $2323.3^{b}$ & $1720.0^{a b}$ & $352.0^{a b}$ & $257.0^{a}$ & $223.0^{a}$ \\
\hline $20 \mathrm{mmol} \mathrm{NaCl} \mathrm{L}-1$ & $1213.7^{a}$ & $2309.6^{b}$ & $2516.0^{a b}$ & $482.7^{b}$ & $437.2^{a b}$ & $456.7^{a b}$ \\
\hline $40 \mathrm{mmol} \mathrm{NaCl} \mathrm{L}-1$ & $3366.7^{b}$ & $2396.7^{b}$ & $3430.0^{b}$ & $1031.3^{c}$ & $1240.7^{c}$ & $763.3 b^{c}$ \\
\hline $80 \mathrm{mmol} \mathrm{NaCl} \mathrm{L}{ }^{-1}$ & $1888.4^{a b}$ & $3383.3^{b}$ & $2085.3^{a b}$ & $1307.2^{c}$ & $1026.3^{b c}$ & $1378.0^{d}$ \\
\hline
\end{tabular}

* Medias con distintas letras difieren significativamente (Tukey HSD, P<0.05).

\section{Crecimiento y biomasa de las plantas}

Las plantas de guanábana no mostraron diferencias significativas en altura entre tratamientos en la fecha 1 ( $F=2.16, p=0.078)$ (Tabla 5). Mientras que en la fecha 2, la altura fue mayor en el testigo que en los tratamientos con 40 y $80 \mathrm{mmol}$ $\mathrm{NaCl} \mathrm{L}^{-1}(\mathrm{~F}=6.95, \mathrm{p}=0.000$; Tukey HSD 5.70 y $6.48, p=0.001$ y 0.000 , y 0.027 ). En la fecha 3 se tuvieron las mismas diferencias $(F=5.88, p=$ 0.000; Tukey HSD 4.56 y $6.06, p=0.02$ y 0.000 , 
Tabla 5. Altura y número de hojas en plantas después de iniciar los tratamientos con agua a distintas concentraciones

\begin{tabular}{|c|c|c|c|c|c|c|c|c|c|}
\hline \multirow{2}{*}{$\begin{array}{l}\text { Tratamiento } \\
\text { /fecha }\end{array}$} & \multicolumn{3}{|c|}{ Guanábana } & \multicolumn{3}{|c|}{ Mango } & \multicolumn{3}{|c|}{ Naranja } \\
\hline & $32 \mathrm{~d}$ & $62 d$ & $93 \mathrm{~d}$ & $32 d$ & $62 d$ & $93 \mathrm{~d}$ & $32 d$ & $62 d$ & $93 \mathrm{~d}$ \\
\hline \multicolumn{10}{|l|}{ Altura $(\mathrm{cm})$} \\
\hline Testigo & $71.56^{a}$ & $81.72^{c}$ & $85.50^{c}$ & $73.62^{a}$ & $74.15^{a}$ & $74.34^{a}$ & $52.85^{a}$ & $61.76^{b}$ & $69.11^{c}$ \\
\hline $10 \mathrm{mmol} \mathrm{NaCl} \mathrm{L}^{-1}$ & $70.32^{a}$ & $78.42^{b c}$ & $82.54^{c}$ & $69.93^{a}$ & $70.79^{a}$ & $69.42^{a}$ & $51.71^{a}$ & $55.37^{a b}$ & $58.14^{b}$ \\
\hline $20 \mathrm{mmol} \mathrm{NaCl} \mathrm{L}^{-1}$ & $68.60^{a}$ & $77.16^{a b c}$ & $81.39^{a b c}$ & $69.10^{a}$ & $70.00^{a}$ & $72.64^{a}$ & $55.75 a$ & $61.00^{b}$ & $63.54^{b c}$ \\
\hline $40 \mathrm{mmol} \mathrm{NaCl} \mathrm{L}^{-1}$ & $66.92^{a}$ & $73.21^{a b}$ & $76.82^{a b}$ & $70.58^{a}$ & $69.71^{a}$ & $71.71^{a}$ & $51.67 \mathrm{a}$ & $55.05^{a b}$ & $53.64^{a b}$ \\
\hline $\begin{array}{l}80 \text { mmol } \mathrm{NaCl} \mathrm{L}^{-1} \\
\mathrm{n} \text { hojas }\end{array}$ & $68.54^{a}$ & $72.05^{a}$ & $73.96^{a}$ & $68.75^{a}$ & $73.92^{a}$ & $67.66^{a}$ & $53.67^{a}$ & $52.74^{a}$ & $53.29^{a}$ \\
\hline Testigo & $18.04^{a}$ & $22.79^{a}$ & $22.00^{a}$ & $19.79^{a}$ & $18.32^{a}$ & $17.71^{b}$ & $57.54^{a b}$ & $57.37^{b}$ & $57.29^{b}$ \\
\hline $10 \mathrm{mmol} \mathrm{NaCl} \mathrm{L}^{-1}$ & $15.67^{a}$ & $21.84^{a}$ & $28.43^{a}$ & $18.13^{a}$ & $16.47^{a}$ & $18.79^{b}$ & $66.25^{b}$ & $59.68^{b}$ & $59.86^{b}$ \\
\hline $20 \mathrm{mmol} \mathrm{NaCl} \mathrm{L}^{-1}$ & $16.88^{a}$ & $23.16^{a}$ & $27.43^{a}$ & $17.67^{a}$ & $18.11^{a}$ & $20.64^{b}$ & $59.38^{a b}$ & $58.72^{b}$ & $57.57^{b}$ \\
\hline $40 \mathrm{mmol} \mathrm{NaCl} \mathrm{L}-1$ & $17.08^{a}$ & $22.26^{a}$ & $27.86^{a}$ & $17.21^{a}$ & $16.16^{a}$ & $18.36^{b}$ & $48.17^{a}$ & $47.42^{a}$ & $37.07^{a}$ \\
\hline $80 \mathrm{mmol} \mathrm{NaCl} \mathrm{L}{ }^{-1}$ & $15.92^{a}$ & $15.21^{a}$ & $20.21^{a}$ & $17.13^{a}$ & $16.37^{a}$ & $12.36^{a}$ & $49.42^{a}$ & $37.79^{a}$ & $20.86^{a}$ \\
\hline
\end{tabular}

*Medias con distinta letra difieren significativamente (Tukey HSD, $\mathrm{P}<0.05$ ).

Tabla 6. Biomasa de hojas, raíces, y total de plantas de guanábana, mango y naranja, a los $93 \mathrm{~d}$ con tratamientos de concentraciones de sal.

\begin{tabular}{|c|c|c|c|c|c|c|c|c|c|c|c|c|c|c|c|}
\hline \multirow[b]{2}{*}{$\mathrm{T}$} & \multicolumn{3}{|c|}{ Raíz (g) } & \multicolumn{3}{|c|}{ Hoja (g) } & \multicolumn{3}{|c|}{ 3PST (g) } & \multicolumn{3}{|c|}{$\mathrm{RPH}(\mathrm{g})$} & \multicolumn{3}{|c|}{ RPR (g) } \\
\hline & G & M & $\mathrm{N}$ & G & $M$ & $\mathrm{~N}$ & G & $M$ & $\mathrm{~N}$ & G & $M$ & $\mathrm{~N}$ & G & $M$ & $\mathrm{~N}$ \\
\hline 1 & 9.75 & 11.18 & $11.20^{b}$ & $4.57^{c}$ & 8.67 & 4.86 & 24.63 & 36.08 & 25.41 & $0.19^{b}$ & 0.24 & 0.19 & 0.40 & 0.31 & 0.44 \\
\hline 2 & 11.22 & 10.19 & $10.42^{a b}$ & $4.87^{c}$ & 7.35 & 5.58 & 28.24 & 33.18 & 25.84 & $0.17^{b}$ & 0.19 & 0.21 & 0.40 & 0.33 & 0.41 \\
\hline 3 & 8.09 & 11.55 & $12.25^{b}$ & $3.34^{a b c}$ & 9.33 & 5.12 & 22.19 & 36.20 & $27.83^{b}$ & $0.16^{b}$ & 0.26 & 0.18 & 0.36 & 0.32 & 0.44 \\
\hline 4 & 7.58 & 7.84 & $9.37^{a b}$ & $4.01^{b c}$ & 10.05 & 2.57 & 21.31 & 33.05 & 18.38 & $0.19^{b}$ & 0.31 & 0.12 & 0.36 & 0.24 & 0.53 \\
\hline 5 & 9.12 & 6.79 & $7.60 \mathrm{a}$ & $0.77^{a}$ & 3.91 & 1.98 & 20.90 & 22.45 & $17.16^{a}$ & $0.03^{a}$ & 0.17 & 0.10 & 0.44 & 0.30 & 0.48 \\
\hline
\end{tabular}

* Medias con distinta letra difieren sifnificativamente (Kruskal - Wallis, $\mathrm{P}<0.05$ ). PST $=$ peso seco total, RPH $=$ peso relativo de las hojas, $\mathrm{RPR}=$ peso relativo de las raíces, $\mathrm{T}=$ tratamiento, $\mathrm{T} 1=$ testigo, $\mathrm{T} 2=10 \mathrm{mmol} \mathrm{NaCl} \mathrm{L}-1, T 3=20 \mathrm{mmol} \mathrm{NaCl} \mathrm{L}-1, \mathrm{~T} 4=\mathrm{mmol}$ $\mathrm{NaCl} \mathrm{L}-1, \mathrm{~T} 5=$ mmol $\mathrm{NaCl} \mathrm{L}-1$.

y 0.018). Mientras que en el mango, no se encontraron diferencias significativas en altura de planta entre tratamientos (Tabla 5).

El número de hojas no mostró diferencias significativas entre fechas. Pero si se detectaron diferencias en la fecha 3 entre el testigo y el tratamiento con $40 \mathrm{mmol} \mathrm{NaCl} \mathrm{L}{ }^{-1}$ que tuvo el mayor número de hojas. En naranja agria, la altura de las plantas no tuvo diferencias significativas entre tratamientos en la fecha $1(F=1.268, p=0.287)$. Mientras que en la fecha 2 las plantas fueron más altas fueron el testigo y el tratamiento con $20 \mathrm{mmol}$ $\mathrm{NaCl} \mathrm{L}^{-1}(\mathrm{~F}=4.557, \mathrm{p}=0.002$, Tukey HSD $=$ 4.85 y $4.44, p=0.008$ y 0.019). En la fecha 3 se mantuvieron las diferencias entre el testigo, y los tratamientos con $20 \mathrm{mmol}$ y $80 \mathrm{mmol} \mathrm{NaCl} \mathrm{L}{ }^{-1}$ (F $=7.216, p=0.000$, Tukey HSD $=6.28$ y $4.07, p$ $=0.000$ y 0.042 ), mientras que las plantas testigo fueron más altas que las plantas del tratamientos con $10 \mathrm{mmol}$ y $40 \mathrm{mmol} \mathrm{NaCl} \mathrm{L}{ }^{-1}$ (Tukey HSD
$=4.35$ y $6.14, p=0.025$ y 0.001 ). El número de hojas en la fecha uno fue mayor en el tratamiento con $10 \mathrm{mmol} \mathrm{NaCl} \mathrm{L}{ }^{-1}$ que en los tratamientos con $40 \mathrm{mmol}$ y $80 \mathrm{mmol} \mathrm{NaCl} \mathrm{L}-1$ ( $\mathrm{F}=5.836, \mathrm{p}$ $=0.000$, Tukey HSD $=5.85$ y $5.44, p=0.001$ y 0.002 ). Mientras que en la fecha 2 , se encontraron más hojas en el testigo, que en el tratamiento con $80 \mathrm{mmol} \mathrm{NaCl} \mathrm{L}{ }^{-1}$ ( $F=5.694, p=0.000$; Tukey $\mathrm{HSD}=4.95$, 5.54 y 5.30 , con $\mathrm{p}=0.006,0.002$ y $0.003)$. Estas diferencias estuvieron presentes en la fecha $3(F=12.97, p=0.000$; Tukey HSD $=7.69$, 8.23 y $7.75 ; p=0.000)$. El testigo y los tratamientos con $10 \mathrm{mmol}$ y $20 \mathrm{mmol} \mathrm{NaCl} \mathrm{L}{ }^{-1}$ tuvieron más hojas que el tratamiento con $40 \mathrm{mmol} \mathrm{NaCl} \mathrm{L}-1$ (Tukey HSD = 4.27, 4.81 y 4.33; $p=0.029,0.010$ y 0.026) (Tabla 5). En la guanábana se observó un incremento de la fecha 1 a la 3 en el número de hojas en los tratamientos con alta CE, mientras que en naranja agria se observó una pérdida de hojas en la fecha 3 en los tratamientos con 40 y $80 \mathrm{mmol}$ 
$\mathrm{NaCl} \mathrm{L}{ }^{-1}$, y en mango fue el tratamiento con 80 mmol NaCl L-1 (Tabla 5).

\section{Biomasa}

No se encontraron diferencias significativas entre los tratamientos para la biomasa de la raíz, hojas, PRH, PRR y el peso seco total en ninguna de las especies evaludas en las primeras 2 fechas. Mientras que en la fecha 3, la biomasa de la raíz de las plantas de naranja agria fue menor en el tratamiento con $80 \mathrm{mmol} \mathrm{NaCl} \mathrm{L}{ }^{-1}$ que en el testigo y el tratamiento con $20 \mathrm{mmol} \mathrm{NaCl} \mathrm{L}{ }^{-1}$ (F $=5.501, \mathrm{p}=0004$; Tukey HSD 4.737 y 6.122, $\mathrm{p}$ $=0.024$ y 0.003$)$. La biomasa del tallo no varió de forma significativa entre los tratamientos en las especies estudiadas. Para la naranja agria no se encontraron diferencias significativas en el peso de hojas entre tratamientos. En guanábana se observó mayor biomasa de las hojas en el testigo y en el tratamiento con $10 \mathrm{mmol} \mathrm{NaCl} \mathrm{L}{ }^{-1}$, que en los otros tratamientos (Kruskal-Wallis K 15.49, p = 0.004; Mann-Whitney, $p=0.012, p=0.021$, y $p=$ 0.037 ). En mango y naranja agria hubo diferencias significativas entre tratamientos $(F=4.263$, con $p$ $=0.029$ en mango; $F=3.525$, con $p=0.025$ en naranja agria). Sin embargo, la comparación entre pares de tratamientos no tuvo valores significativos. La biomasa total no varió entre tratamientos en guanábana y mango en la fecha $3(\mathrm{~F}=2.464, \mathrm{p}=$ 0.078 ; y $F=2.58, p=0.069$ ). Aunque en naranja agria se encontraron diferencias significativas $(F=$ $3.525, p=0.025)$. No se encontraron diferencias significativas entre tratamientos en el peso de raíces en mango y naranja agria, ni iferencias significativas en el peso de las hojas entre especies.

\section{Modelo}

La biomasa total de las plantas en la fecha 3 se relacionó con la CE de los tratamientos mediante las siguientes ecuaiones:

\footnotetext{
$Y_{r(\text { guanabana })}=0.9292 e^{-0.03555 * C E}$

$Y_{r(\text { mango })}=1.250 e^{-0.08973 * C E}$

$Y_{r(\text { naranja })}=1.0661 e^{-0.07899 * C E}$
}

$Y_{r}$ es la biomasa de las diferentes especies y CEi es la CE de los tratamientos. Los coeficientes de correlación $(r)$ de los modelos fueron de 0.73 , 0.99 y 0.84 , para guanábana, mango y naranja agria, respectivamente. A partir de dichos modelos se infiere que la naranja agria reduce su biomasa en $50 \%$ a una CE de $9580 \mu \mathrm{S} \mathrm{cm}{ }^{-1}$, el mango a 10 $220 \mu \mathrm{S} \mathrm{cm}^{-1}$ y la guanábana a $17430 \mu \mathrm{S} \mathrm{cm}{ }^{-1}$. El índice de tolerancia al estrés (ST-Index) fue de 16810,9300 y $8830 \mu \mathrm{S} \mathrm{cm}^{-1}$ para guanábana, mango y naranja agria, respectivamente.

\section{DISCUSIÓN}

Salinidad y composición de las especies en los huertos de la costa del estado de Tabasco

Se observó una amplia variación en la CE en los primeros $30 \mathrm{~cm}$ del suelo. Los valores más altos se presentaron en un huerto cercano a la laguna Mecoacán, lo que probablemente se deba a las corrientes de agua salada que entran a la laguna. La CE en este huerto fue mayor a los $3500 \mu \mathrm{S} \mathrm{cm}^{-1}$ al inicio de la temporada de secas, y de $2000 \mu \mathrm{S} \mathrm{cm}^{-1}$ a finales de la época de lluvias. La CE es mayor en el suelo, debido a que la cantidad de agua en el suelo es menor que en la suspensión usada para medir la CE. Al respecto, Oosterbaan (2003) estima que la CE en el agua del suelo saturado (CE) es 10 veces la concentración medida en suspensiones 1 : 5 . Por lo que suelos con CE entre 4000 y $8000 \mu \mathrm{S} \mathrm{cm}^{-1}$ se consideran como ligeramente salinos. En la Figura 2 , se observa que los suelos de cuatro huertos tienen valores superiores.

En promedio, la CE fue mayor a principios de la temporada de secas y menor a finales de la época de lluvias. Esto se esperaba, debido a que el agua de lluvia, al percolarse a través del suelo, arrastra las sales al subsuelo. La variación en la CE entre huertos al inicio de la temporada de secas, puede tener relación con la textura del suelo, ya que los suelos arcillosos acumulan mayor cantidad de sal, que los suelos arenosos, al retener mayor cantidad de agua e incrementar su capacidad de intercambio catiónico (Palma y Triano 2007). En los suelos arenosos y sueltos, la capacidad de intercambio 


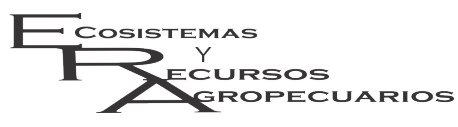

catiónico es menor, y en consecuencia el suelo retiene menor cantidad de iones de $\mathrm{Na}^{+}$. Aunado a ello, el agua de lluvia se drena en mayor cantidad en estos suelos por la acción de la gravedad, lixiviando las sales disueltas.

EI ACC muestra relación entre la composición de especies y la conductividad del suelo. Lo que significa que en algunos huertos las concentraciones de sal alcanzan niveles que no todas las especies toleran. En las condiciones de los huertos, las plantas se exponen a periodos de sequía, lo que ocasiona que la concentración de sal en el agua del suelo aumente a niveles mayores que en suelos saturados de agua, lo que ocasiona desequilibrios nutricionales, debido a los altos potenciales osmóticos en el suelo (Kopittke 2012, Schröder 2013). La ausencia o escasez de elementos esenciales y la mayor presencia de $\mathrm{Na}^{+}$y $\mathrm{Cl}^{-}$limitan el crecimiento y productividad de las plantas (Ali et al. 2014). Lo que implica, que al aumentar la salinidad en los suelos de los huertos, la composición de especies y la multifuncionalidad sean afectadas.

La profundidad del agua freática no mostró relación con la composición de especies. Las raíces de las plantas, al concentrarse en la capa superior del suelo, absorben en primera instancia el agua de la lluvia, evitando que el agua salina del manto freático influya en el crecimiento de las plantas. La salinidad del agua freática sólo afecta en situaciones donde se encuentra a escasa profundidad, permitiendo que la sal suba a la capa superior del suelo por acción capilar, o bien ocupando los poros del suelo.

\section{Tolerancia a la salinidad de tres especies}

Las mediciones de CE en la fecha 2 muestran una ligera salinidad en los tratamientos con $10 \mathrm{mmol}$ y $20 \mathrm{mmol} \mathrm{NaCl} \mathrm{L}{ }^{-1}$, y una moderada salinidad en los tratamientos con 40 y $80 \mathrm{mmol} \mathrm{NaCl}$ $\mathrm{L}^{-1}$. En la fecha 3, la salinidad fue moderada en los tratamientos con 10 y $20 \mathrm{mmol} \mathrm{NaCl} \mathrm{L}{ }^{-1}$, y alta en los tratamientos con $40 \mathrm{mmol}$ y $80 \mathrm{mmol} \mathrm{NaCl} \mathrm{L}{ }^{-1}$ (Tabla 3). Las mediciones produjeron algunos resultados inesperados, donde la CE disminuyó a pesar de haber aplicado agua con mayor CE. Lo anterior,
Alcudia-Aguilar et al. Salinidad, composición y crecimiento de frutales Esosist. Recur. Agropec. 4(10):1-12,2017

puede deberse a la distribución heterogénea de la sal en el suelo (Bazihizina et al. 2012) o que al tomar la muestra no se homogeneizó lo suficientemente el suelo, lo que pudo afectar la CE. La CE fue $100 \%$ mayor en el suelo de la mitad inferior de las macetas que en el suelo de la mitad superior (Tabla 4). En la mitad inferior de las macetas, las condiciones fueron de alta salinidad en los tratamientos con $10,20,40$ y $80 \mathrm{mmol} \mathrm{NaCl} \mathrm{L}^{-1}$, mientras que en la mitad superior, las condiciones fueron no salinas en el testigo y el tratamiento con $10 \mathrm{mmol} \mathrm{NaCl}$ $\mathrm{L}^{-1}$. Lo anterior, implica que las plantas pueden explorar diferencialmente el suelo y aprovechar la variación en la salinidad, absorbiendo los nutrientes de las partes menos salinas. Las raíces expuestas a bajas concentraciones de sal aprovechan los elementos disponibles $\left(\mathrm{K}^{+}\right.$y $\left.\mathrm{Ca}^{++}\right)$, lo que le permite a la planta incrementar su biomasa, para evadir el efecto de la salinidad. Este mecanismo puede jugar un papel en guanábana y el mango, cuya biomasa seca total no varió entre tratamientos (Tabla 6). Posiblemente la naranja agria no tiene esta capacidad de compensación. Lo anterior, confirma la flexibilidad y capacidad de algunas especies de regular su exposición a los efectos de la salinidad (Bazihizina et al. 2012).

En general, las especies difieren en sus mecanismos de respuesta a la salinidad (Bharti et al. 2013), algunas responden produciendo numerosas hojas y eliminando la sal mediante la defoliación (Carter y Nippert 2012). Otras especies reaccionan de manera diferencial con el $\mathrm{pH}$ del suelo (CalvoPolanco et al. 2014), mientras que el jitomate reduce la salinidad mediante la producción de compuestos de glicina betaína y la trehalosa (Rivero et al. 2014). Los resultados en el número de hojas y su biomasa indican que la eliminación mediante la defoliación puede jugar un papel en la guanábana, mientras que en naranja agria y mango no se observaron indicios de que opere este mecanismo. Posiblemente esto se relaciona con los índices de tolerancia al estrés (ST-Index) encontrados. La naranja agria fue la especie menos tolerante (8.83 $\left.\mathrm{dS} \mathrm{m}^{-1}\right)$, seguida del mango $\left(9.30 \mathrm{dS} \mathrm{m}^{-1}\right)$ y la

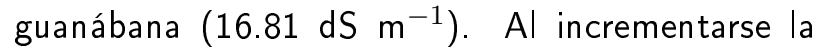


salinidad en los huertos en la costa, se reducirá el área de distribución de la naranja agria.

\section{CONCLUSIONES}

La salinidad del suelo influyó en la composición de especies arbóreas y arbustivas en los huertos familiares. La mayor salinidad se presentó a principios de la temporada de secas y la menor a principio de los nortes. De las tres especies evaluadas, la naranja agria es la especie menos tolerante a la salinidad; el mango tiene tolerancia intermedia, y la guanábana es la especie más tolerante. Los resultados indican que el incremento de la salinidad impactará en la composición de las especies de los huertos en la costa del estado de Tabasco. Ante la problemática asociada a la salinidad y sus efectos. Se recomienda investigar los mecanismos de adaptación de las plantas a condiciones de mayor salinidad, así como los mecanismos de las prácticas de cultivo.

\section{AGRADECIMIENTOS}

Al Consejo Nacional de Ciencia y Tecnología (CONACYT) por el financiamiento del proyecto "Gestión y estrategias de manejo sustentable para el desarrollo regional en la cuenca hidrográfica transfronteriza Grijalva" (clave 143303) y becó al primer autor para cursar la Maestría en Ciencias en Recursos Naturales y Desarrollo Rural en El Colegio de la Frontera Sur. Al proyecto PROMEP CA SEP "Balance de agua y uso de suelo en 3 condiciones ambientales".

\section{LITERATURA CITADA}

Ali S, Charles TC, Glick BerR (2014) Amelioration of high salinity stress damage by plant growth-promoting bacterial endophytes that contain ACC deaminase. Plant Physiology and Biochemistry 80: 160-67.

Bazihizina N, Barrett-Lennard EG, Colmer TD (2012) Plant growth and physiology under heterogeneous salinity. Plant and Soil 354: 1-19.

Bharti N, Barnawal D, Awasthi A, Yadav A, Kalra A (2013) Plant growth promoting rhizobacteria alleviate salinity induced negative effects on Growth, Oil Content and Physiological Status in Mentha Arvensis. Acta Physiologiae Plantarum 36: 45-60.

Caetano E, Innocentini, V, Magaña V, Martins S, Méndez B (2010) Cambio climático y el aumento del nivel del mar. En: Botello AV, Villanueva-Fragoso S, Gutiérrez J, Rojas Galaviz JL (ed). Vulnerabilidad de las zonas costeras mexicanas ante el cambio climático. SEMARNAT-INE, UNAM, Universidad Autónoma de Campeche. pp: 238 - 304.

Calvo-Polanco M, Alejandra-Equiza M, Señorans J, Zwiazek JJ (2014) Responses of rat root (Raf.) plants to salinity and $\mathrm{pH}$ conditions. Journal of Environmental Quality 43: 578 - 86

Carter JM, Nippert JB (2012) Leaf-level physiological responses of Tamarix ramosissima to increasing salinity. Journal of Arid Environments 77: 17-24

Chaitanya KV, Krishna CHR, Ramana GV, Beebi SKK (2014) Salinity stress and sustainable agriculture a review. Agricultural Reviews 35: 34-41

Clair SBSt, Lynch JP (2010) The opening of Pandora's Box: climate change impacts on soil fertility and crop nutrition in developing countries. Plant and Soil 335: 101-115.

CONAGUA (2012) Pronóstico del tiempo. Consejo Nacional del Agua. Servicio Meteorológico Nacional, México. http://smn.conagua.gob. $\mathrm{mx} /$ index.php?option=com_content\&view=article\&id=48. Fecha de consulta 25 de junio del 2012. 
Correia PJ, Gama F, Pestana M, Martins-Loucao MA (2010) Tolerance of young (Ceratonia siliqua L.) carob rootstock to $\mathrm{NaCl}$. Agricultural Water Management 97: 910-916.

Etchevers BJD (1992) Manual de métodos para análisis de suelos, plantas, aguas y fertilizantes. Análisis rutinarios en estudios y programas de fertilidad. En: Laboratorios de fertilidad, Centro de Edafología (ed). Colegio de Postgraduados. Montecillo, Estado de México. pp: 12-16

Galluzzi G, Eyzaguirre P, Negri V (2010) Home gardens: neglected hotspots of agro-biodiversity and cultural diversity. Biodiversity and Conservation 19: 3635-3654

Gliessman SR (1998) Agroecology: Ecological processes in sustainable agriculture. Ann Arbor Press, Chelsea, MI, USA. En: Méndez VE, Lok R (ed). Interdisciplinary analysis of home gardens in Nicaragua: micro - zonation plant use y socioeconomic importance. Agroforestry Systems. pp: 85 - 96.

Hammer $\varnothing$, Harper DAT, Ryan PD (2001) PAST: Paleontological statistics software package for education y data analysis. Paleontología Electrónica 4: 1-9.

Idohou R, Fandohan B, Salako VK, Kassa B, Gbédomon RC, Yédomonhan H, et al. (2014) Biodiversity conservation in home gardens: traditional knowledge, use patterns and implications for management. International Journal of Biodiversity Science, Ecosystem Services and Management 10: 89-100

Jelgersma S, Zijp M, Van Der, Brinkman R (1993) Sea level rise by the coastal lows in the developing world. Journal of Coastal Research 9: 958-972.

Kopittke PM (2012) Interactions between Ca, Mg, Na and K: Alleviation of toxicity in saline solutions. Plant and Soil 352: 353-362.

Kopittke PM, Blamey FPC, Kinraide TB, Wang P, Reichman SM, Menzies NW (2011) Separating multiple, short-term deleterious effects of saline solutions to the growth of cowpea seedlings. New Phytologist 189: $1110-1121$

Kumar BM, Nair PKR (2004) The enigma of tropical home gardens. Agroforestry Systems 61: 135 - 152.

Nakashima DJ, McLean KG, Thulstrup H, Castillo AR, Rubis J (2012) Weathering uncertainty: Traditional knowledge for climate change assessment and adaptation. Paris, UNESCO, and Darwin,UNU. http://www.ipmpcc.org/2012/06/13/weathering-uncertainty-traditional-knowledge-for-climate-changeassessment-and-adaptation/. Fecha de consulta 5 de octubre de 2014.

Oosterbaan RJ, Nijland HJ (2003) Determining the saturated hydraulic conductivity. In: Ritzema HP (ed). Drainage principles and applications. International Institute for Land Reclamation and Improvement (ILRI), Publication 16, Second revised edition, Wageningen, the Netherlans. pp: 1-38.

Ortíz-Pérez MA, Hernández-Santana JR, Figueroa JM, Gama L (2010) Tasas del avance transgresivo y regresivo en el frente deltaico tabasqueño: en el periodo comprendido del año 1995 al 2008. En: Botello AV, Villanueva-Fragoso S, Gutiérrez J, Rojas Galaviz JL (ed). Vulnerabilidad de las zonas costeras mexicanas ante el cambio climático. SEMARNAT-INE, UNAM-ICMyL, UAC. Campeche, México. pp: $305-324$.

Palma LDJ, Triano SA (2002) Plan de uso sustentable de los suelos de Tabasco, Vol. II. Ed. Colegio de Postgraduados-ISPROTAB. Villahermosa, Tabasco. México. 160p.

Pitman MG, Läuchli A (2002) Global impact of salinity and agricultural ecosystems. In: Läuchli A, Lüttge U (ed) Salinity: environment - plants - molecules. Kluwer Academic, Dordrecht. pp: 3-20.

Remison SU, Iremiren GO, Y Thomas GO (1998) Effect of salinity on nutrient content of the leafs of coconut seedlings. Plant y Soil 109: 135-138. 
Rivero RM, Mestre TC, Mittler R Rubio F, Garcia-sanchez F, Martinez V (2014) The combined effect of salinity and heat reveals a specific physiological, biochemical and molecular response in tomato plants. Plant, Cell \& Environment 37: 1059-1073.

Schröder N, Lazarovitch N, Vanderborght J, Vereecken H, and Javaux M, (2013) Linking Transpiration Reduction to Rhizosphere Salinity Using a 3D Coupled Soil-Plant Model. Plant and Soil 377: 277-93.

Steppuhn H, Van Genuchten MTH, Gireve CM (2005a) Root-zone salinity. II. Indices for tolerance in agricultural crops. Crop Science 45: 221-232.

Steppuhn H, Van Genuchten MTH, Grieve CM (2005b) Root-zone salinity. I. Selecting a product-yield index y response function for crop tolerance. Crop Science 45: 209-220.

Xu HM, Tam NFY, Zan QJ, Bai M, Shin PKS, Vrijmoed LLP, et al. (2014) Effects of salinity on anatomical features and physiology of a semi-mangrove plant Myoporum bontioides. Marine Pollution Bulletin 85: 738-746.

Zhao K, Song J, Feng G, Zhao M, Liu J (2011) Species, types, distribution, and economic potential of halophytes in China. Plant and Soil 342: 495-09.

Zhu Z, Chen J, Zheng HL (2012) Physiological and proteomic characterization of salt tolerance in a mangrove plant, Bruguiera gymnorrhiza (L.) Lam. Tree Physiology 3: 1378-1388 